\title{
Endoscopic ultrasound-guided radiofrequency ablation for hepatocellular carcinoma in cirrhosis: a case report test for efficacy and future perspectives $\square$
}

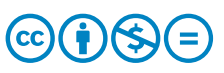

\author{
Authors \\ Gianpiero Manes ${ }^{1,2}$ \\ Institutions \\ 1 Gastroenterology and Digestive Endoscopy Unit, ASST \\ Rhodense, Garbagnate Milanese, Milan, Italy \\ 2 Gastroenterology and Digestive Endoscopy Unit, ASST \\ Rhodense, Rho, Milan, Italy \\ 3 Gastroenterology and Digestive Endoscopy Unit, AORN \\ Cardarelli, Naples, Italy \\ 4 Digestive Endoscopy Unit, Fondazione Policlinico \\ Universitario A. Gemelli IRCCS, Catholic University, \\ Rome, Italy
}

Germana de Nucci ${ }^{1}$, Cristina della Corte ${ }^{2}$, Raffaella Reati ${ }^{1}$, Nicola Imperatore ${ }^{3}$, Ilaria Arena ${ }^{2}$, Alberto Larghi $^{4}$,

submitted 27.4.2020

accepted after revision 15.7 .2020

Bibliography

Endoscopy International Open 2020; 08: E1713-E1716

DOI 10.1055/a-1236-3105

ISSN 2364-3722

(C) 2020. The Author(s).

This is an open access article published by Thieme under the terms of the Creative Commons Attribution-NonDerivative-NonCommercial License, permitting copying and reproduction so long as the original work is given appropriate credit. Contents may not be used for commecial purposes, or adapted, remixed, transformed or built upon. (https://creativecommons.org/licenses/by-nc-nd/4.0/)

Corresponding author

Germana de Nucci, MD, Gastroenterology and Digestive

Endoscopy Unit, ASST Rhodense, Viale Forlanini 98, 0024,

Garbagnate Milanese, Milan, Italy

Fax: +39 02994302905

germanadenucci1@gmail.com

\section{ABSTRACT}

Background The percutaneous approach allows for effective and safe treatment of liver lesions. But in case of subcapsular or left segments location, this approach seems to be less effective or unsafe. Endoscopic ultrasound-guided radiofrequency ablation (EUS-RFA) is a new technique used to treat pancreatic and neuroendocrine tumors in patients unfit for surgery.

Methods Hereby, we describe the case of a 70-year-old patient with cirrhosis with a large subcapsular hepatocellular carcinoma (HCC) in II-III-IVb segments, in which surgery or percutaneous therapies were not feasible, treated with EUS-RFA. The HCC was treated using an EUS-RFA (EUSRA) system, which consists of a $19 G$ water-cooled monopolar RFA needle and a dedicated generator system.

Results After a multidisciplinary discussion, the lesion was ablated in two different sessions, which resulted in destruction of about $70 \%$ of neoplastic tissue. A second step surgery was required but initially refused by the patient.

Conclusions EUS-RFA could be an effective way to treat left hepatic lesions not manageable with conventional percutaneous methods. This case report does not highlight concerns about safety of this approach and this observation needs to be validated in a larger cohort of patients with cirrhosis.

\section{Introduction}

Hepatocellular carcinoma (HCC) is a growing health problem with increased incidence rates globally. The most frequent risk factors are hepatitis C/hepatitis B virus (HCV/HCB) infections, alcohol, and metabolic diseases [1]. According to a recent standard of care, single HCC nodules with a diameter $<5 \mathrm{~cm}$ or multifocal nodules $(<3)$ can be treated by resection or transplantation [2], but in patients with a single HCC nodule who are unfit for surgery due to portal hypertension, comorbidities or previous surgery, a locoregional approach with radiofrequency ablation (RFA) can be chosen with good results. RFA is based on the principle that high-frequency alternating current is converted into thermal energy, producing a coagulative necrosis useful in the treatment of different cancers [3]. In treatment of HCC, the application of percutaneous RFA can be limited by presence of large interposing vessels, obesity or disadvantageous location of the lesions in the liver (subcapsular/left 


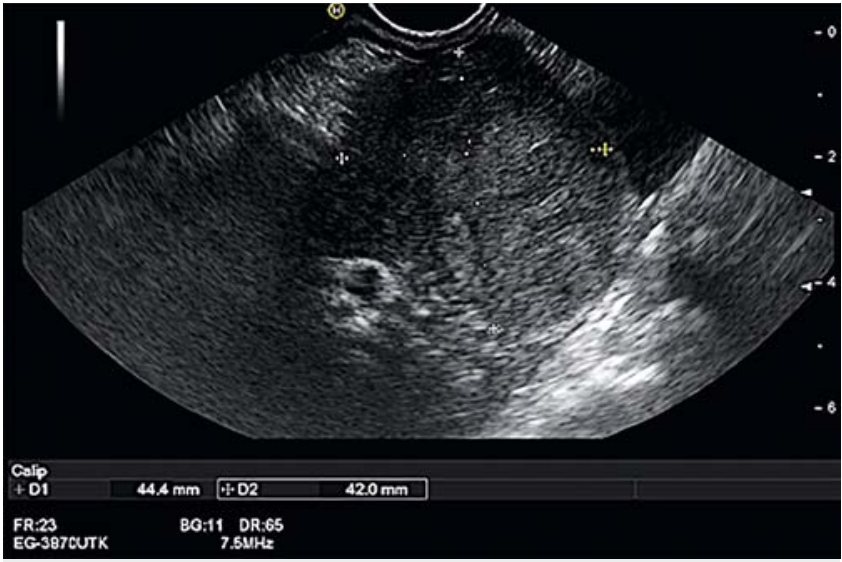

Fig. 1 EUS image of HCC.

lobe/caudate segment) [4]. These latter limitations can be overcome by a newly developed RFA needle to be used under endoscopic ultrasound (EUS) guidance. EUS-RFA has been successfully used to treat pancreatic neuroendocrine tumors [5]. Moreover, because the left liver lobes lie in strict contiguity with the stomach, EUS-guided RFA (EUS-RFA) has been described in one case report in which the lesion could not be approached percutaneously [6]. We describe the second case of EUS-RFA of a liver lesion occupying the left liver lobes.

\section{Case report}

A 70-year-old male presented to our clinic with HCV-related liver cirrhosis (Child-Pugh A6, MELD 9). A $30 \times 45 \mathrm{~mm}$ focal lesion localized at II-III and IVb liver segment was detected with abdominal ultrasound and confirmed to be highly suspicious for an $\mathrm{HCC}$ with contrast-enhanced computed tomography (CT) scan. Blood tests showed albumin $3.7 \mathrm{mg} / \mathrm{dL}$, bilirubin $0.9 \mathrm{mg} /$ $\mathrm{dL}$, international normalized ratio 1.29 , platelets $81.000 / \mu \mathrm{L}$, liver enzymes about two to three times the upper limit of normal (ULN), and serum HCV-RNA titer 1.314.860 UI/mL. Physical exam showed mild peripheral edema, which was treated with hyposodic diet and diuretics. At a multidisciplinary meeting, the lesion was considered borderline for surgical resection, which was refused by the patient. Percutaneous RFA and microwave ablation (MWA) were not considered technically feasible because of the subdiaphragmatic location of the nodule and risk of a too large necrosis in the presence of initial signs of portal hypertension, as demonstrated by the low platelet count and splenomegaly on ultrasound exam. For all these reasons, EUS-guided RFA therapy of the nodule was proposed to the patient, who accepted and signed informed consent.

EUS-RFA was performed 4 weeks later using deep sedation with propofol and a therapeutic linear EUS endoscope (EG3870UTK; Pentax Germany). At the level of the distal part of the stomach, under the lesser gastric curve, the entire left liver lobe was visualized and the lesion detected, which appeared as a $45 \times 48 \mathrm{~mm}$ hypoecogenic nodule with sharp borders ( $\triangleright$ Fig. 1) and typical HCC behavior at contrast-enhanced EUS (CH-EUS) after intravenous injection of Sonovue (Bracco Inter-

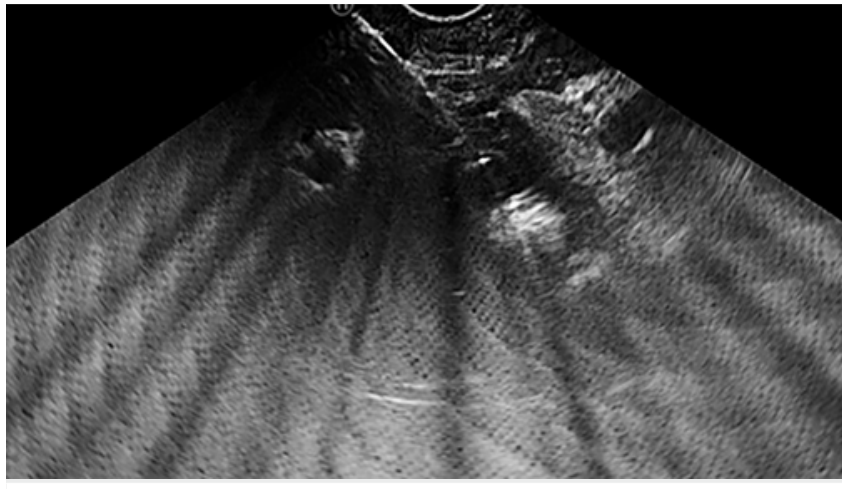

Fig. 2 HCC EUS-RFA with presence of typical steam bubbles.

national B.V., Amsterdam, Netherland). EUS-RFA was performed us a 19G water-cooled monopolar RFA needle (EUSRA, EUS-guided RFA electrode), $150 \mathrm{~cm}$ long with a 10 -mm active tip, and a generator system (VIVA combo) (TaeWoong Medical, Korea). The system features an internal cooling system that circulates chilled saline solution through the needle electrode during the RFA procedure to prevent charring of the surface of the electrode and to improve the accuracy of ablation. The lesion was punctured at different sites, and $30 \mathrm{~W}$ ablation power was delivered at each site under direct EUS control. The tip of the needle was positioned in the distal periphery of the lesion and moved proximally to ablate the whole lesion, including a few millimeters of normal tissue surrounding the nodule. The ablation was stopped when the tissue impedance measured in real time by the generator suddenly increased. Indeed, an impedance rise, meaning reduced capacity of the tissue to conduct electricity, is an indirect sign of cellular disruption, tissue charring, and hampering of electrical conduction. Overall, 15 overlapping applications of RFA, each 10 to $15 \mathrm{sec}$ onds in duration, were performed to cover the entire nodule volume. Necrosis of the tissue was confirmed by the appearance of echogenic bubbles in an area surrounding the needle tip ( $\mathbf{F i g . 2 , ~}$ - Video 1). The completeness of treatment was assessed with repeat $\mathrm{CH}$-EUS, which was able to demonstrate absence or persistence of residual vital tissue ( $\triangleright$ Fig. 3 ).

After a recovery time of 1 hour, the patient was transferred back to the ward, where his pain score and blood pressure were measured every 3 hours and blood testing was done at 3, 12, and 24 hours.

The patient tolerated the procedure well and had no symptoms or clinical decompensation. The Child Pugh score worsened temporarily to $\mathrm{B} 9$ and he had mild fever ( $\max 37.7^{\circ} \mathrm{C}$ ) and an increase in $\mathrm{C}$-reactive protein to four times the upper limit of normal.

Two weeks later, antiviral therapy with sofosbuvir plus velpatasir $(400 / 100 \mathrm{mg})$ was started and produced a prompt virological response with serum HCV RNA suppression. A CT scan performed 1 month later showed a reduction in lesion size of about $50 \%$ ( Fig.4). A repeat treatment was scheduled and performed with the same technique in September 2019. The second treatment was again well tolerated and a CT scan per- 

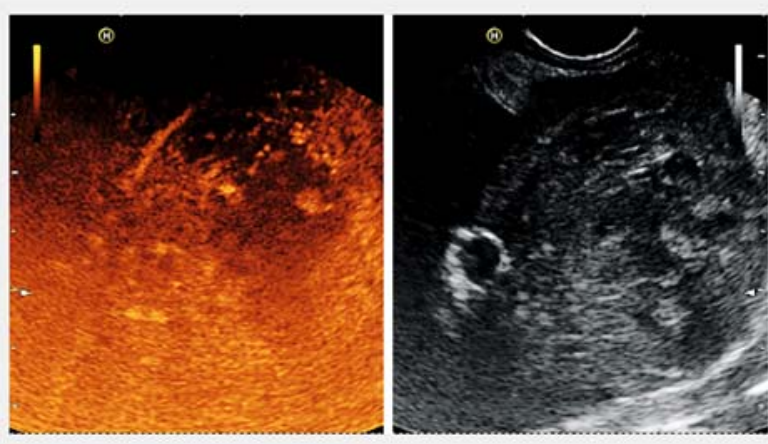

- Fig. 3 CH-EUS control after first RFA showing effective central necrosis area as a result of the ablation.

formed 1 month later showed a further reduction in the lesion size, however, there was a vital neoplastic tissue remnant at segment IVb of about $15 \times 20 \mathrm{~mm}$ diameter. With this decrement in the lesion size, liver resection was a consideration and the patient agreed and underwent an atypical left lobe liver resection without complications in January 2020. Histology revealed the presence of HCC with a grade of differentiation G3 without vascular/lymphatic/neural invasion with a necrotic coagulative area of about $70 \%$ as a result of the previous EUS-RFA treatments. The surgical margins were free from neoplasia and the final pathologic classification following the AJCC (American Join Committee on Cancer) $8^{\text {th }}$ Edition was T1b, N0. The patient was discharged after an uneventful hospitalization 3 weeks later with full liver compensation. His HCV relapsed 30 days later and a new antiviral treatment has been planned to start very soon.

\section{Discussion}

We report the second case of EUS-RFA treatment of a $30 \times 45$ mm HCC lesion localized to the II-III and IVb liver segments. In the first report by Attili et al., a 2-cm lesion of the III hepatic segment was completely ablated without any adverse events (AEs) [6]. In our case, the treatment was well tolerated and only a transient mild liver decompensation occurred after both RFA sessions, causing a transient change in Child Pugh score from $A 6$ to $B 9$, which is an expected consequence of ablative treatments that result in necrosis of pathological tissue in the liver. The response to RFA treatment, due to the larger size of the lesion and its location in the IV b segment, was incomplete, but allowed the patient's disease to be downstaged so that he could undergo liver resection, which was otherwise considered a risky procedure, and maintain good performance status (Child A6 pre surgery). Moreover, the patient was able to get access to antiviral therapy, which would not have been indicated if the nodule was left untreated.

Novel procedures usually carry potential benefits and several uncertainties. Several conditions may hinder the applicability of percutaneous RFA, the first of which is obesity, which is very prevalent in the United States and Europe. HCC nodules located

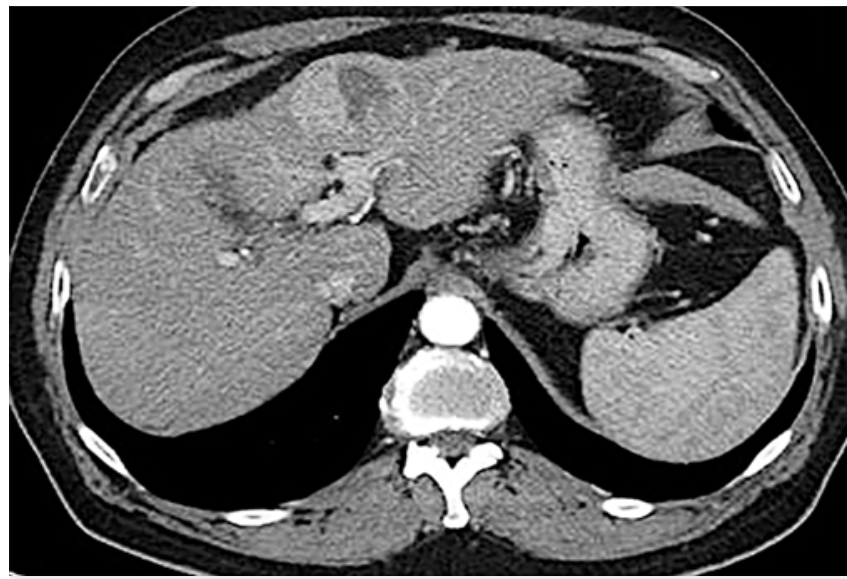

- Fig. 4 CT control after second EUS-RFA treatment showing a 20-mm periferal vital remnant at the IVb segment.

in the left lobe and in the caudate segment may be not easily approachable percutaneously. Moreover, the subcapsular/subdiaphragmatic location may carry an intrinsic risk of thermal damage, resulting in pleural effusion or hemothorax and a higher risk of tumor seeding/tumor recurrence [7]. In such cases, video-laparoscopic RFA may be an alternative to percutaneous treatment, but it is likely to be associated with a higher rate of adverse events related to increased surgical risk in patients with cirrhosis, and to anatomical difficulties in patients with portal hypertension or previous abdominal surgery [8]. In our case, the EUS approach allowed us to perform RFA for a lesion that would have not been otherwise manageable in a conventional way. A further benefit offered by the EUS approach has been its capability to better identify the vascular structures surrounding the nodule to reduce risk of thermal energy dispersion.

Besides documenting the technique's advantages, our case also raises questions about EUS-RFA. First, previous experience with the VIVA generator system in the treatment of liver lesions is very limited [6]. We decided to set the generator at a power of $30 \mathrm{~W}$ after testing the ablation capacity of the system on bovine livers (unpublished data). Noteably, this setting has already been used to treat pancreatic lesions (20-50 W) [9].

A second critical issue regards the assessment of necrosis while performing RFA. An indirect sign of the development of necrosis in our case was represented by the appearance of echogenic bubbles within the lesion. Because echogenic steam occurs when the tissue temperature increases to more than $100^{\circ} \mathrm{C}$, development of bubbles involving the whole lesion and its borders can be interpreted as achievement of the maximal thermal effect on the tissue. The absence of vital tissue was confirmed in our patient by performing $\mathrm{CH}$-EUS with Sonovue. However, we observed a significant discrepancy between the presumptive complete necrosis of the lesion as assessed at $\mathrm{CH}$-EUS and the persistence of vital tissue at contrast-enhanced CT scan performed 30 days later. Vilana et al. [10] reported that $\mathrm{CH}$-EUS performed more than 24 hours after treatment has very low sensitivity (27.3\%) for identifying vital neoplastic tis- 


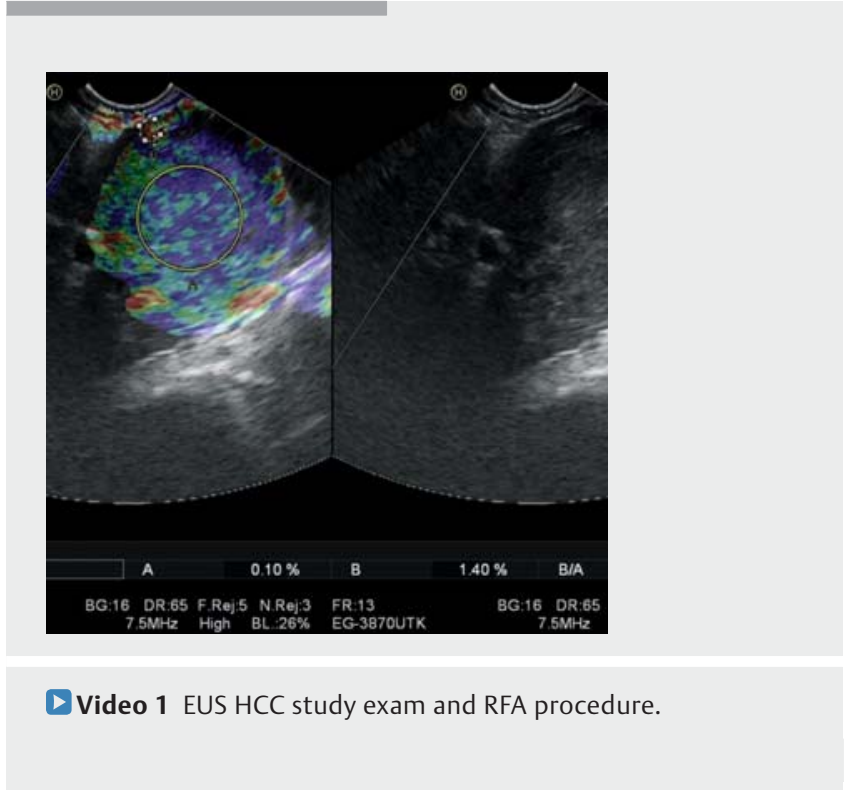

sue when compared to CT scan performed 1 month after the procedure. This is probably due to the fact that steam bubbles that develop during treatment may obscure part of the lesion, making it very difficult to differentiate residual neoplastic tissue from reactive hyperemia around the nodule. This effect is likely to be more evident in cases of very large lesions and for those located away from the EUS probe, such as in our case. In general, contrast-enhanced EUS is per se less sensitive than contrast-enhanced CT for assessing exact nodule extension. Possible disagreement in enhancement patterns between sonography/EUS and CT/magnetic resonance imaging contrast agents may explain such a discrepancy, but this aspect has to be studied in the future to improve real-time assessment of efficacy of the RFA procedure after lesion ablation.

\section{Conclusions}

In conclusion, our case suggests that EUS-RFA could be an effective modality for treating hepatic lesions that are not otherwise manageable with the percutaneous route with no early or late AEs and no negative impact on subsequent surgery. Our observations, however, need to be validated in larger series of patients with cirrhosis who have lesions located in the left EUS-approachable segments.

Competing interests

The authors declare that they have no conflict of interest.

\section{References}

[1] Jemal A, Bray F, Center MM et al. Global cancer statistics. CA Cancer ] Clin 2011; 61: 69-90

[2] Galle Peter $R$ et al. EASL Clinical Practice Guidelines: Management of hepatocellular carcinoma. J Hepatology 2018; 69: 182-236

[3] McDermott S, Gervais DA. Radiofrequency ablation of liver tumors. Semin Intervent Radiol 2013; 30: 49-55

[4] Peng ZW, Lin X], Zhang Y] et al. Radiofrequency ablation versus hepatic resection for the treatment of hepatocellular carcinomas $2 \mathrm{~cm}$ or smaller: a retrospective comparative study. Radiology 2012; 262: 1022-1033

[5] Pai M, Habib N, Senturk $\mathrm{H}$ et al. Endoscopic ultrasound guided radiofrequency ablation, for pancreatic cystic neoplasms and neuroendocrine tumors. World J Gastrointest Surg 2015; 7: 52-9

[6] Attili F, Boskiski I, Bove $V$ et al. EUS-guided radiofrequency ablation of a hepatocellular carcinoma of the liver. VideoGIE 2018; 3: 149-150

[7] Berzigotti A, Seijio S, Reverter E et al. Assessing portal hypertension in liver diseases. Exp Rev Gastroenterol Hepatol 2013; 2: 141-155

[8] Seror O, Haddar D, N'Kontchou G et al. Radiofrequency ablation for the treatment of liver tumors. JVIR 2005; 12: 981-990

[9] Scopelliti F, Pea A, Conigliaro R et al. Technique, safety, and feasibility of EUS-guided radiofrequency ablation in unresectable pancreatic cancer. Surg Endosc 2018; 3: 4022-4028

[10] Vilana R, Bianchi L, Varela M et al. Is microbubble-enhanced ultrasonography sufficient for assessment of response to percutaneous treatment in patients with early HCC? Eur Radiol 2006; 16: 24542462 